\title{
Quasar variability measurements with SDSS repeated imaging and POSS data
}

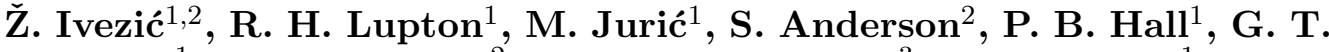 \\ Richards $^{1}$, C. M. Rockosi ${ }^{2}$, D. E. Vanden Berk ${ }^{3}$, E. L. Turner ${ }^{1}$, G. R. \\ Knapp $^{1}$, J.E. Gunn ${ }^{1}$, D. Schlegel ${ }^{1}$, M.A. Strauss ${ }^{1}$ and D.P. Schneider ${ }^{4}$ \\ ${ }^{1}$ Princeton University, Princeton, USA \\ ${ }^{2}$ University of Washington, Seattle, USA \\ ${ }^{3}$ University of Pittsburgh, Pittsburgh, USA \\ ${ }^{4}$ The Pennsylvania State University, University Park, USA
}

\begin{abstract}
We analyze the properties of quasar variability using repeated SDSS imaging data in five UV-to-far red photometric bands, accurate to $0.02 \mathrm{mag}$, for $\sim 13,000$ spectroscopically confirmed quasars. The observed time lags span the range from 3 hours to over 3 years, and constrain the quasar variability for rest-frame time lags of up to two years, and at rest-frame wavelengths from $1000 \AA$ to $6000 \AA$. We demonstrate that $\sim 66,000$ SDSS measurements of magnitude differences can be described within the measurement noise by a simple function of only three free parameters. The addition of POSS data constrains the long-term behavior of quasar variability and provides evidence for a turn-over in the structure function. This turn-over indicates that the characteristic time scale for optical variability of quasars is of the order 1 year.
\end{abstract}

\section{Rest-frame Time Lag and Wavelength, and Luminosity Dependence}

Significant progress in the description of quasar variability has been recently made by employing SDSS data. Vanden Berk et al. (2004, hereafter VB) used a combination of imaging and spectrophotometric magnitudes to investigate the correlations of variability with rest frame time lag (up to 2 years), luminosity, rest wavelength, redshift, the presence of radio and X-ray emission, and the presence of broad absorption line outflows. Variability on longer time scales was studied by de Vries, Becker \& White (2003, hereafter dVBW) who compared SDSS and POSS measurements. We extend these studies by analyzing repeated SDSS imaging data. Here we summarize the main results obtained for a sample of $\sim 13,000$ quasars with $\sim 66,000$ magnitude difference measurements in 5 SDSS bands $\dagger$ (for a detailed description of SDSS survey see Stoughton et al. 2001, and references therein), and recalibrated POSS I and POSS II photometry (Sesar et al. 2004). Details and supporting analysis will be presented elsewhere (Ivezić et al. 2004, in prep).

We find that SDSS magnitude difference measurements, $\Delta m$, follow an exponential distribution, $p(\Delta m) \propto \exp \left(-|\Delta m| / \Delta_{c}\right)$, where the characteristic variability scale, $\Delta_{c}$ is a function of rest-frame time lag $\left(\Delta t_{R F}\right.$, days), wavelength $\left(\lambda_{R F}, \AA\right)$, and absolute magnitude in $i$ band $\left(M_{i}\right)$. The variability scale is related to the more commonly used structure function (see dVBW for definition) by $S F=\sqrt{2} \Delta_{c}$; we find that the function

$$
S F=(1.00 \pm 0.03)\left[1+(0.024 \pm 0.04) M_{i}\right]\left(\Delta t_{R F} / \lambda_{R F}\right)^{0.30 \pm 0.05}
$$

describes $\Delta m$ measurements to within the measurement noise ( $\sim 0.02$ mag). Note that there is no dependence on redshift (see Figure 1). The difference between the data and this model (right panel) reveals a feature at $\sim 2800 \AA$, which is coincident with the MgII line. This anti-correlation between the continuum and $\mathrm{Mg}$ II line variability is indeed known

$\dagger$ We do not employ spectrophotometric magnitudes as they are 3-4 times less accurate than imaging magnitudes, and are not available for $u$ and $z$ bands. 

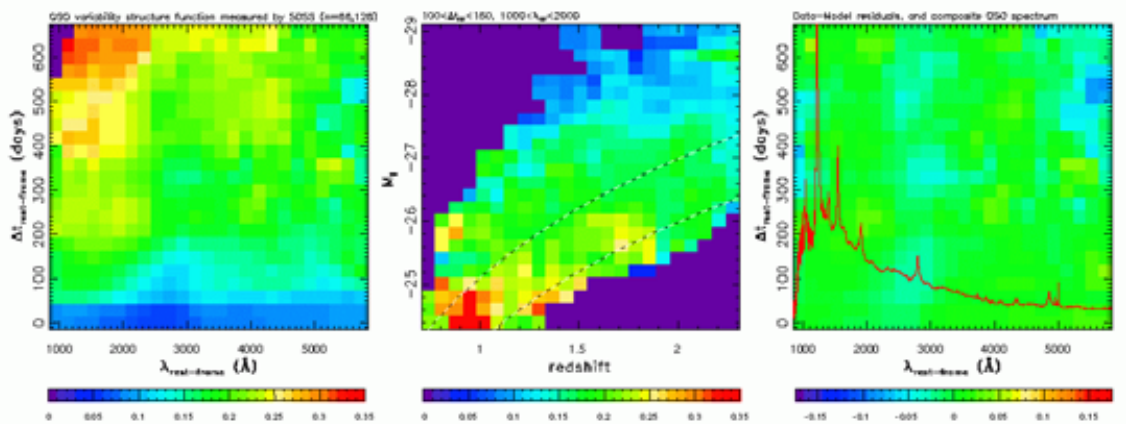

Figure 1. The left panel displays the measured structure function as a function of rest-frame time lag and wavelength (each pixel contains $\sim 25$ objects). The middle panel shows the structure function in a narrow range of rest-frame time lag and wavelength $\left(100 \mathrm{~d}<\Delta t_{R F}<150 \mathrm{~d}\right.$, $1000 \AA<\lambda_{R F}<2000 \AA$ ), as a function of redshift and luminosity. The lines of constant variability are nearly parallel to the redshift axis, suggesting that the dependence of variability on luminosity is much stronger than the dependence on redshift. The right panel displays the difference between the data shown in the left panel and the best-fit model described by eq.(1.1). The red line shows the composite quasar spectrum from Vanden Berk et al. (2001). The negative residuals at $\sim 2800 \AA$ are due to the anti-correlation of continuum and MgII line variability.

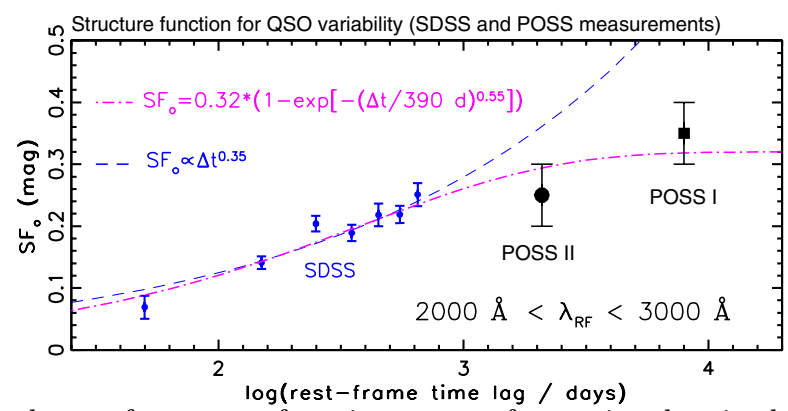

Figure 2. The dependence of structure function on rest-frame time lag, in the wavelength range 2000-3000 $\AA$, for two data sets: SDSS-SDSS for short time lags (small symbols), and SDSS-POSS for long time lags (large symbols).

from reverberation mapping - due to the excellent SDSS photometry, we recovered this result using broad-band data!

\section{Detection of a Turn-over in the Structure Function}

The time lags between Palomar Observatory Sky Surveys (POSS I and POSS II) and SDSS are much longer (up to $\sim 50$ years), than spanned by the available SDSS repeated imaging data, and thus offer a possibility of detecting deviations from the simple power-law measured for short time scales using repeated SDSS imaging. The observed SDSS-POSS long-term variability is smaller than predicted by the extrapolation of the power-law from eq. 1.1 (Sesar et al. 2004). The observations (Fig. 2) are well described by

$S F\left(\Delta t_{R F}\right)=D\left(1-\mathrm{e}^{-\left(\Delta t_{R F} / \tau\right)^{\gamma}}\right)$ with $D=0.32 \pm 0.03, \tau=(390 \pm 80)$ days, and $\gamma=0.55 \pm 0.05$. This best-fit is shown in Figure 2 by the dot-dashed line. We conclude that the characteristic time scale for optical variability of quasars is of the order 1 year.

\section{References}

de Vries, W. H., Becker, R. H., \& White, R. L. 2003, AJ, 126, 1217

Sesar, B., et al. 2004, astro-ph/0403319

Vanden Berk, D. E., et al. 2004, ApJ, 601, 692

Vanden Berk, D. E., et al. 2001, AJ, 122, 549

Stoughton, C., et al. 2002, AJ, 123, 485 